\title{
Efficacy of Transcatheter Arterial Chemoembolization Followed by Sorafenib for Intermediate/Advanced Hepatocellular Carcinoma in Patients in Japan: A Retrospective Analysis
}

\author{
Takamasa Ohki $^{1} \cdot$ Koki Sato $^{1} \cdot$ Mari Yamagami $^{1} \cdot{\text { Daisaku } \text { Ito }^{1}}^{1}$ \\ Tomoharu Yamada $^{1}$ - Koki Kawanishi ${ }^{1} \cdot$ Kentaro Kojima $^{1}$. \\ Michiharu Seki $^{1} \cdot$ Nobuo Toda ${ }^{1} \cdot$ Kazumi Tagawa $^{1}$
}

Published online: 8 October 2015

(C) The Author(s) 2015. This article is published with open access at Springerlink.com

\begin{abstract}
Background Sorafenib might prevent hepatocellular carcinoma (HCC) recurrence caused by the promotion of neoangiogenesis after transarterial chemoembolization (TACE).

Objectives To evaluate the efficacy and safety of TACE followed by sorafenib for treating advanced HCC.

Patients and Methods We retrospectively analyzed 95 advanced HCC patients treated with TACE between July 2008 and December 2012 at our institution. Twenty-four patients received TACE followed by sorafenib within 14 days (S-TACE) and 71 received TACE alone. Progression-free survival (PFS) and cumulative survival from the time of non-responsiveness to TACE were compared between groups and predictive factors for PFS were analyzed.

Results The median patient age was 72.2 years and 74 patients were male $(77.9 \%)$. Although median tumor size was similar between groups, the mean tumor number was significantly higher in the S-TACE versus TACE-alone group (16 vs. $8, P=0.04$ ). The number of prior treatments was significantly higher in the S-TACE group. Other baseline variables were similar. There were two severe adverse events in the S-TACE group and none in the TACE-alone group. Median PFS (189 vs. 106 days, $P=0.02)$ and median overall survival time (861 vs. 467 days, $P=0.01$ ) from the time of non-responsiveness to TACE were significantly longer with S-TACE than TACE alone. Adjusting for significant factors in univariate
\end{abstract}

Takamasa Ohki anb72547@nifty.com

1 Department of Gastroenterology, Mitsui Memorial Hospital, Kanda-Izumi-cho 1, Chiyoda-ku, Tokyo 101-8643, Japan analysis, multivariate analysis indicated that sorafenib administration, tumor size, and alanine transaminase were independent predictors of PFS.

Conclusion TACE followed by sorafenib significantly improved PFS and survival in patients with advanced HCC unresponsive to TACE.

\section{Key Points}

TACE followed by sorafenib improved PFS and survival from time of non-responsiveness to TACE in patients with advanced HCC.

HCC patients treated with S-TACE had a higher median number of tumors than did those treated with TACE alone.

Independent factors for increased survival were the administration of sorafenib, tumor size, and alanine transaminase levels.

\section{Introduction}

Hepatocellular carcinoma (HCC) is the second most common cause of cancer-related death worldwide [1]. Risk factors associated with $\mathrm{HCC}$ include old age and persistent infection with hepatitis B virus (HBV) or hepatitis $\mathrm{C}$ virus (HCV). Patients with HCV-associated liver cirrhosis have a five times higher risk of developing HCC than patients with HBV-associated liver cirrhosis. The annual mortality rate of HCC was approximately 750,000 deaths worldwide in 
2012 [1]. Recent epidemiological studies reported a high prevalence of $\mathrm{HCC}$ in Asia and Africa, which is explained by the high incidence of HBV in these regions [2], and a high prevalence in Japan because of the high incidence of $\mathrm{HCV}$ [3]. However, the incidence of $\mathrm{HCC}$ is also increasing in Western countries [3-5].

Of note, Japan has one of the highest rates of HCC compared with other developed countries [6]. Risk factors for HCC in Japan include older age ( $>40$ years), male sex, having advanced fibrosis, and being positive for viral hepatitis markers [7-9]. The relatively high mean age of the Japanese population and the high number of $\mathrm{HCV}$ carriers might explain why the annual carcinogenesis rate in patients with type $\mathrm{C}$ cirrhosis is higher in Japan (7-8 \%) than in developed Western countries (1-3\%) [10].

In addition to virus-related HCC, non-virus-related liver cirrhosis disorders including nonalcoholic steatohepatitis and alcoholic liver disease are also risk factors for developing HCC. In Japan, approximately 5-20\% of HCC cases are negative for $\mathrm{HBV}$ or $\mathrm{HCV}$ markers, and non-viral associated cases of HCC associated with insulin resistance and fatty liver disease are increasing $[11,12]$.

In Western countries, HCC is diagnosed early in $30-40 \%$ of cases and early treatment prolongs patient survival [13]. In Japan, a nationwide surveillance program for HCC has been initiated and $65 \%$ of HCC patients are detected at early stages allowing curative surgical treatment [14].

Currently, there is a lack of effective systemic therapy for the treatment of advanced HCC, and therefore patients who present with advanced HCC are difficult to cure and have a poor prognosis. Although there is an initial beneficial response in $\mathrm{HCC}$ patients receiving surgery, the recurrence rate of HCC after surgery is $60 \%$ over 5 years $[15,16]$. The standard first-line treatment for advanced HCC is transcatheter arterial chemoembolization (TACE), which is frequently performed in HCC patients deemed ineligible for surgery and in patients with HCC recurrence [17]. TACE is used to induce hepatic artery occlusion of HCCs, thus blocking the blood supply to tumor cells and inhibiting tumor growth. TACE is often used in combination with various chemotherapy agents. Previous randomized clinical trials reported that TACE prevented HCC recurrence for up to 6 months after surgery $[18,19]$, and improved survival rates $(1$ year $=82 \%$ and 2 years $=$ $63 \%$ [18]; 1 year $=57 \%, 2$ years $=31 \%$, and 3 years $=$ $26 \%$ [20]).

Despite these findings, some patients are unresponsive to TACE and do not show a prolonged time to progression/ recurrence of HCC [21, 22]. Furthermore, although TACE can influence tumor progression, partial responses were observed in 15-55\% of patients [23], and HCC can recur after TACE $[9,21]$. Despite its repeated use, TACE eventually fails to provide clinical benefit and the patient's status is termed TACE failure/refractoriness, as first described by the Japanese Society of Hepatology (JSH) [21].

Recently, the revised JSH guidelines defined TACE failure as an "insufficient response after $\geq 2$ consecutive TACE procedures that is evident on response evaluation computed tomography or magnetic resonance imaging after 1-3 months, even after chemotherapeutic agents have been changed and/or the feeding artery has been reanalyzed" [24].

Furthermore, TACE is associated with elevated vascular endothelial growth factor (VEGF) concentrations that might have a role in neoangiogenesis and functional liver recovery, and thus encourage repeat tumor growth [25-28]. Therefore, there is a need to enhance the methodology of TACE or identify alternative treatments for advanced HCC.

Sorafenib is a multikinase inhibitor that inhibits the RafMEK-ERK signaling pathway to inhibit tumor cell proliferation and blocks VEGF receptors (VEGFRs) to prevent neoangiogenesis [29]. Sorafenib has been investigated as an anticancer drug with antiproliferative and antiangiogenic properties and was shown to have benefits in preclinical studies of renal cell, hepatocellular, breast, and colorectal carcinomas [27]. It was approved for the treatment of advanced liver cancer in Western countries in 2007 after a phase III trial showed it increased the median overall survival (OS) time in HCC patients (sorafenib vs. placebo: 10.7 vs. 7.9 months; hazard ratio [HR] 0.69, $95 \%$ confidence interval [CI] 0.55-0.87, $P<0.001$ ) [30]. An Asia Pacific phase III trial of HCC patients also showed that sorafenib increased the median OS time (sorafenib vs. placebo: 6.5 vs. 4.2 months, HR $0.68,95 \%$ CI $0.50-$ $0.93, P<0.014)$, increased the time to progression, and was well tolerated [31]. Previous studies of sorafenib in patients who were unresponsive to TACE or when used as maintenance therapy in patients with advanced HCC demonstrated increased OS time and time to progression versus patients who received hepatic arterial infusion chemotherapy using cisplatin [32], patients who continued TACE [33], or those receiving placebo [34].

A Japanese retrospective comparative study demonstrated no statistically significant difference in the OS time between HCC patients who received sorafenib or TACE monotherapy [35]. The findings of the Japanese study by Nishikawa et al. [35] are in line with reports of previous studies using a combined concurrent administration of TACE and sorafenib in the USA [28, 36], China [37, 38], and Germany [39]. Other studies have demonstrated the benefit of sequential therapy of TACE followed by sorafenib on prolonging the time from TACE to disease progression in Italy [40], and TACE with interrupted dosing of sorafenib in China/Korea/Taiwan [41]. 
Meta-analyses of combined TACE and sorafenib treatment have reported conflicting data. A systematic review and meta-analysis of combined TACE and sorafenib trials by Liu et al. [42] indicated that combination therapy might increase time to progression but not OS time in unresectable HCC patients when compared with TACE alone. However, another systematic review and meta-analysis by Yang et al. [43] suggested that the combined treatment improved OS time, time to progression, and treatment responses compared with TACE alone.

Although the administration of sorafenib following TACE might prevent HCC recurrence caused by the promotion of neoangiogenesis by TACE-induced neoangiogenesis, it is currently unclear whether the use of TACE followed by sorafenib provides additional survival benefits to HCC patients compared with TACE alone. Therefore, this retrospective study evaluated the efficacy and safety of TACE followed by sorafenib in Japanese patients with advanced HCC who became non-responsive to TACE.

\section{Methods}

\subsection{Patients}

In this retrospective study, 95 patients with advanced HCC treated with TACE were enrolled between July 2008 and December 2012 at the Department of Gastroenterology, Mitsui Memorial Hospital, Tokyo, Japan. The patients were followed up until December 2014. The inclusion criteria included HCC patients, some of whom were previously non-responsive to TACE [experiencing progressive disease (PD) at least twice with conventional TACE]. PD was defined according to the modified Response Evaluation Criteria In Solid Tumors (RECIST) criteria for HCC [44]. Exclusion criteria included patients of Child-Pugh class C, those under 20 years of age, pregnancy, or those with an allergy to sorafenib.

Of the 95 patients, 24 patients who were previously nonresponsive responsive to TACE were treated with TACE followed by sorafenib (S-TACE group), and 71 patients who were responsive to TACE were treated with TACE alone (TACE-alone group). Patients were divided into groups depending on whether they became TACE unresponsive during the follow-up period. Patients attended follow-up every month, which consisted of blood tests including tumor makers, and contrast-enhanced computed tomography (CT) or magnetic resonance imaging (MRI) every $2-4$ months. Thus, patients who were TACE-unresponsive were administered sorafenib from January 2010 onwards. Because sorafenib use was started from August 2009 in our institute, the duration of treatment was shorter in some cases in the S-TACE group.
The oral administration of sorafenib was started within 2 weeks after TACE because TACE induced the transient elevation of liver enzymes and fever. The starting dose of sorafenib was $400 \mathrm{mg} /$ day. If patient side effects were acceptable after 1 week of administration as assessed by the Common Terminology Criteria for Adverse Events v4.0 (CTCAE) $<$ grade 2 , the dosage was increased to $600 \mathrm{mg}$. If patient side effects were acceptable even after the dosage was increased to $600 \mathrm{mg}$ for 1 week, the dosage was finally increased to $800 \mathrm{mg}$.

We compared the progression-free survival (PFS) and OS time between the TACE and S-TACE groups and determined the factors associated with PFS.

Written informed consent was obtained from all patients when they began treatment for HCC, but was not required for the present analyses. The study protocol followed all appropriate guidelines according to the Declaration of Helsinki. As this was a retrospective study, institutional ethics committee approval was not required.

\subsection{Diagnosis of Hepatocellular Carcinoma (HCC)}

HCC diagnosis was based on typical findings on imaging studies such as arterial hyperattenuation and portal hypoattenuation by contrast-enhanced dynamic CT or gadolinium ethoxybenzyl diethylenetriamine pentaacetic acid MRI.

\subsection{Transarterial Chemoembolization (TACE) Procedure}

TACE was performed on an inpatient basis. First, a 4-Fr modified Shepherd-hook catheter and a 4-Fr hepatic-curve catheter were placed in the celiac artery, through the bilateral femoral arteries, according to Seldinger's method. Digital subtraction angiography was performed from the celiac artery to evaluate hepatic artery anatomy. A micro-catheter was inserted through the 4-Fr catheter and placed in the proper or common hepatic artery for hepatic arteriography. The procedure used $3.0 \mathrm{~mL}$ of contrast medium, $30 \mathrm{mg}$ of doxorubicin (Adriacin; Kyowa Hakko Kirin, Tokyo, Japan), and $3.0 \mathrm{~mL}$ of iodized oil (Lipiodol Ultra-Fluid; Guerbet Japan, Tokyo, Japan) or Milipratin $70 \mathrm{mg}$ with $4.0 \mathrm{~mL}$ of iodized oil or CDDP $100 \mathrm{mg}$ and $10.0 \mathrm{~mL}$ of iodized oil. The amounts of contrast medium and iodized oil in this suspension were arbitrarily adjusted according to tumor size. This agent was injected into each feeder artery of the HCC, followed by an infusion of gelatin sponge particles.

\subsection{Study Endpoints}

The primary endpoint of the study was PFS in the TACEalone and S-TACE groups. The secondary endpoint was 
the survival time from the point of non-responsiveness to TACE in patients with HCC.

\subsection{Adverse Events}

CTCAE was used to determine the severity of adverse events, which were classified as grade I to IV [45]. Adverse events were collected from the patients' medical records.

\subsection{Statistical Analysis}

Categorical variables were compared by Fisher's exact test. Continuous variables were compared using the unpaired Student's $t$ test. Each survival rate was estimated using the Kaplan-Meier method comparing values with the log-rank test. To elucidate the risk factors that affected PFS, we tested the same variables used in regression analysis obtained at the time of entry by univariate Cox's proportional hazard regression analysis. Multivariate Cox's proportional hazard regression analysis was also performed to assess the risk for PFS with variables that had a $P$ value $<0.05$ in the univariate analyses. Statistical analyses were performed using StatView version 5.0. A $P$ value of $<0.05$ was considered statistically significant (Fig. 1).

\section{Results}

The mean age of patients was 72.2 years and was not significantly different between the S-TACE and TACEalone groups (70.0 vs. 72.9 years). Previous treatments administered to HCC patients included TACE (mean 1.6 times), radiofrequency ablation (mean 2.2 times), and resection in 11 patients. Overall, 74 patients were male

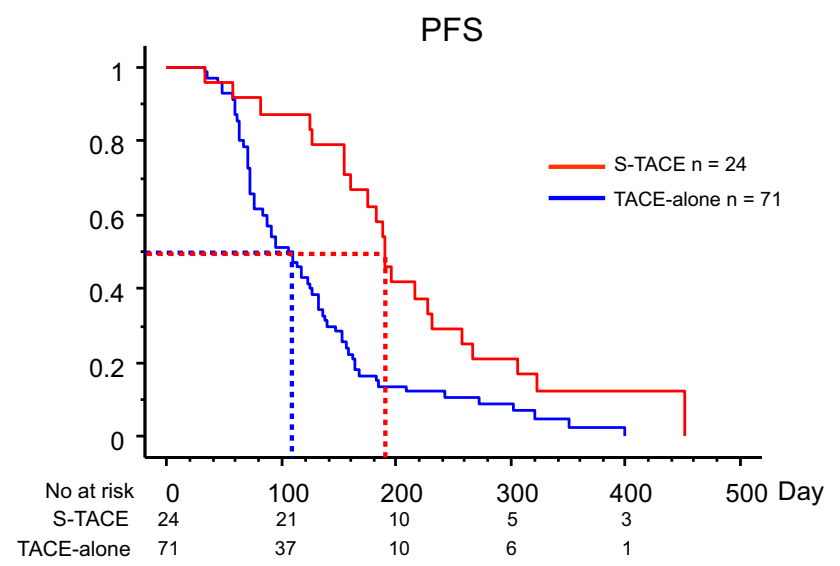

Fig. 1 Progression-free survival. The median progression-free survival is shown as a dotted line $* P<0.01$ compared with S-TACE. TACE transarterial chemoembolization, S-TACE TACE followed by sorafenib
$(77.9 \%)$ (Table 1). Of note, most patients had intermediate HCC (stage I: $n=3$; stage II: $n=30$; stage III: $n=55$; stage IVa: $n=3$; and stage IVb: $n=4)$. The S-TACE group included significantly more patients with HCC stage III, IVa, or IVb than did the TACE-alone group $(P=0.046)$ (Table 1). Although the median tumor size was $25 \mathrm{~mm}$ in the S-TACE group and $23 \mathrm{~mm}$ in the TACE-alone group $(P=0.91)$, the median number of tumors was significantly greater in the S-TACE group (7 vs. $4, P<0.01$ ). The median number (range) of prior treatments was also significantly higher in the S-TACE group than in the TACE-alone group in terms of both TACE $(2[1-3]$ vs. $1[0-2], P<0.01)$ and radiofrequency ablation (4 [1-5] vs. 1 [0-3], $P<0.01)$. Liver function, blood biochemistry, and tumor markers were not significantly different between the two groups (Table 2). The primary endpoint was median PFS, which was significantly longer in the S-TACE group $(n=24)$ than in the TACEalone group $(n=71)(189$ vs. 106 days, $P<0.01)$ (Fig. 1). The median survival time in patients unresponsive to TACE was significantly longer in the S-TACE group than in the TACE-alone group (861 vs. 467 days, $P<0.01$ ) (Fig. 2).

After adjusting for relevant confounding factors, Cox hazard proportional multivariate analysis indicated that the administration of sorafenib (HR 0.38, $P<0.01$ ), tumor diameter (per $10 \mathrm{~mm}$, HR 1.12, $P<0.01$ ), and alanine transaminase levels (per $10 \mathrm{IU} / \mathrm{ml}$, HR 1.04, $P<0.01$ ) were independently associated with PFS in patients unresponsive to TACE (Table 3). There were no factors that were independently associated with OS time in patients unresponsive to TACE (data not shown).

Factors that contributed to survival time from the timepoint of non-responsiveness to TACE were administration of sorafenib (HR 0.43, $P<0.01$ ), tumor diameter (per $10 \mathrm{~mm}$, HR 1.15, $P<0.01$ ), and albumin levels (per $1.0 \mathrm{~g} /$ dL, HR 0.53, $P=0.01$ ) (Table 4). Age and sex were included in the univariate analysis but did not retain statistical significance (data not shown); therefore, they were not included in the multivariate analysis. In addition, disease stage was not included in the model because tumor diameter and tumor number, which indicate disease severity, were included.

The majority of adverse events related to sorafenib, as determined by CTCAE version 4 , in the TACE-alone or S-TACE groups were classified as Grade 1 or 2 and included hand foot syndrome, rash, diarrhea, fatigue, and hypertension. There were two severe adverse events in the S-TACE group (Grade 3 liver dysfunction: $n=1$; Grade 4 liver dysfunction: $n=1$ ). There were no serious adverse events, or adverse events greater than grade 2 , in the TACE-alone group. Therefore, the tolerability of TACE was not affected by the addition of sorafenib to the 
Table 1 Patient characteristics

\begin{tabular}{lccc}
\hline Variables & S-TACE $(n=24)$ & TACE alone $(n=71)$ & $P$ value \\
\hline Age (years) & $70.0(65.3-75.2)$ & $72.9(68.7-77.6)$ & 0.07 \\
Male & $20(83.3 \%)$ & $54(76.1 \%)$ & 0.50 \\
HCV-Ab positive & $18(75.0 \%)$ & $48(67.6 \%)$ & 0.61 \\
Child-Pugh class A & $17(70.8 \%)$ & $40(56.3 \%)$ & 0.24 \\
Stage III, IVa, or IVb HCC & $20(83.3 \%)$ & $42(59.2 \%)$ & 0.046 \\
History of liver resection & $4(16.7 \%)$ & $8(11.3 \%)$ & 0.90 \\
History of RFA (median, range) & $4(1-5)$ & $1(0-3)$ & $<0.01$ \\
History of TACE (median, range) & $2(1-3)$ & $1(0-2)$ & $<0.01$ \\
\hline
\end{tabular}

Values are expressed as $n(\%)$ or median (range)

TACE transarterial chemoembolization, $S$-TACE TACE followed by sorafenib, $H C V$ hepatitis $\mathrm{C}$ virus, $H C C$ hepatocellular carcinoma, $R F A$ radiofrequency ablation

Table 2 Laboratory data

\begin{tabular}{lccc}
\hline Variables & S-TACE $(n=24)$ & TACE alone $(n=71)$ & $P$ value \\
\hline ALB $(\mathrm{g} / \mathrm{dL})$ & $3.8(3.6-4.1)$ & $3.6(3.3-4.1)$ & 0.11 \\
ALT $(\mathrm{IU} / \mathrm{L})$ & $65(29-82)$ & $52(24-54)$ & 0.39 \\
T.Bil $(\mathrm{mg} / \mathrm{dL})$ & $1.1(0.8-1.2)$ & $0.8(0.6-1.2)$ & 0.17 \\
Plt $\left(\times 10^{4} / \mu \mathrm{L}\right)$ & $12.4(8.5-14.3)$ & $12.0(8.0-15.3)$ & 0.51 \\
PT $(\%)$ & $74(64-80)$ & $72(62-82)$ & 0.43 \\
AFP $(\mathrm{ng} / \mathrm{mL})$ & $50(18-445)$ & $29(6-223)$ & 0.50 \\
DCP $(\mathrm{mAU} / \mathrm{mL})$ & $195(62-973)$ & $74(28-680)$ & 0.58 \\
\hline
\end{tabular}

Values are expressed as median (range)

$T A C E$ transarterial chemoembolization, $S$-TACE TACE followed by sorafenib, $A L B$ albumin, $A L T$ alanine aminotransferase, $T$. Bil total bilirubin, $P l t$ platelets, $P T$ prothrombin time, $A F P$ alpha-fetoprotein, $D C P$ desgamma-carboxy prothrombin

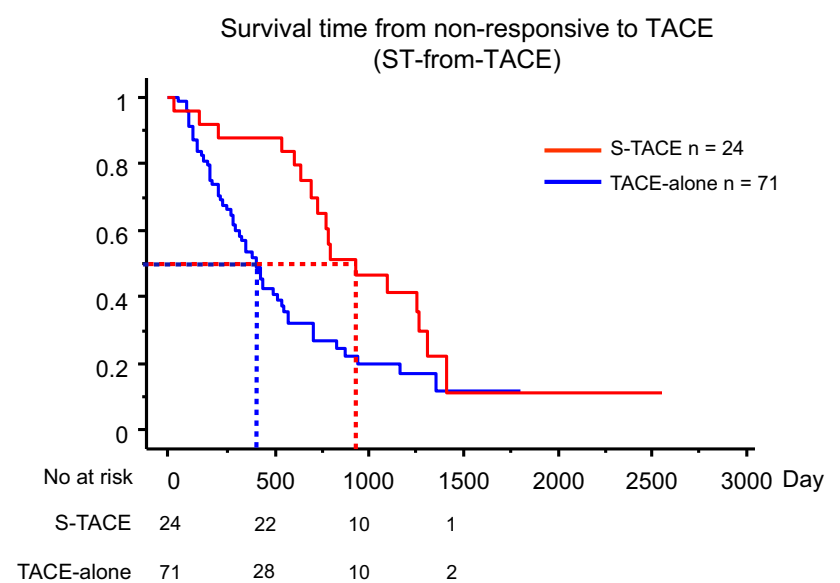

Fig. 2 Overall survival time from non-responsive to TACE. The median overall survival time is shown as a dotted line $* P<0.01$ compared with S-TACE. TACE transarterial chemoembolization, $S$ TACE TACE followed by sorafenib

treatment protocol. In addition, portal or hepatic vein invasion occurred in two patients in the TACE group and in one patient in the S-TACE group.
Table 3 Factors contributing to progression-free survival

\begin{tabular}{lll}
\hline Variable & HR $(95 \% \mathrm{CI})$ & $P$ value \\
\hline Use of sorafenib & $0.38(0.22-0.63)$ & $<0.01$ \\
Tumor size (per 10 mm) & $1.12(1.04-1.20)$ & $<0.01$ \\
ALT (per 10 IU/L) & $1.04(1.01-1.06)$ & $<0.01$ \\
\hline Values are expressed as median (range) & \\
$C I$ confidence interval, $A L T$ alanine aminotransferase, $H R$ hazard \\
ratio
\end{tabular}

Table 4 Factors contributing to overall survival time from non-responsiveness to TACE

\begin{tabular}{llr}
\hline Variable & HR $(95 \% \mathrm{CI})$ & $P$ value \\
\hline Use of sorafenib & $0.43(0.24-0.76)$ & $<0.01$ \\
Tumor diameter (per $10 \mathrm{~mm})$ & $1.15(1.04-1.26)$ & $<0.01$ \\
ALB (per $1.0 \mathrm{~g} / \mathrm{dL})$ & $0.53(0.33-0.87)$ & 0.01 \\
T.Bil $($ per $1.0 \mathrm{mg} / \mathrm{dL})$ & $1.08(0.99-1.17)$ & 0.07 \\
\hline
\end{tabular}

$\overline{T A C E}$ transarterial chemoembolization, $C I$ confidence interval, $A L B$ albumin, T.Bil total bilirubin, $H R$ hazard ratio 
HCC patients in the S-TACE group received oral administration of $400 \mathrm{mg} /$ day sorafenib with a median administration time of 412 days. The sorafenib dose was reduced in three patients $(200 \mathrm{mg} /$ day) and increased in seven patients (600 mg, $N=5 ; 800 \mathrm{mg}, N=2)$. Sorafenib was stopped in one patient because of Grade 4 liver injury and in 11 patients because of disease progression. During the follow-up period, 24 patients received additional TACE.

Patients who only received one TACE before being declared unresponsive in the S-TACE group had developed extra-hepatic metastasis.

\section{Discussion}

This retrospective study investigated the effectiveness, safety, and survival effects of TACE followed by the administration of oral sorafenib in Japanese TACE nonresponder HCC patients and compared them with patients receiving TACE alone. We found that treatment with TACE followed by sorafenib significantly improved the survival time and PFS in patients unresponsive to TACE compared with those receiving TACE alone, and was well tolerated. These findings confirmed those reported by many previous studies using TACE monotherapy in terms of its efficacy and safety [9, 17, 22, 23, 46-48]. In addition, the PFS and time to progression were significantly longer in the S-TACE group compared with the TACE-alone group in the current study, consistent with previous studies of combined TACE plus sorafenib or sequential TACE plus sorafenib in nonresponsive HCC patients [28, 33, 36-41, 46].

Currently, the first-line treatments for intermediate/advanced HCC in Japan include TACE, hepatic arterial infusion chemotherapy (HAIC)/transcatheter arterial infusion (TAI), or sorafenib monotherapy. Although the use of TACE is beneficial in patients with advanced disease, HCC recurs in some patients because the devascularization effect of TACE is transient. Furthermore, the survival benefit of sorafenib decreases over time and the response rates are often poor. It was previously shown that sustained TACE treatment was more likely to cause liver failure than symptomatic treatments. Furthermore, because multiple TACE administration has a negative effect on liver function requiring treatment to be stopped in some patients, this might allow the potential regrowth or metastases of the tumor [20, 49]. Therefore, it is important to determine which patients might respond to multiple TACE treatment to choose the correct treatment modality for specific HCC patients: TACE, sorafenib, combined treatment, or sequential treatment. Moreover, because the repetition of TACE causes a deterioration in liver function, it might have negative effects, meaning that subsequent therapies are necessary [22, 50, 51].

In Japan, the JSH guidelines have shifted from TACE towards other approaches including TAI/HAIC or sorafenib, which is recommended as a standard therapy for patients with intermediate $\mathrm{HCC}$ who are non-responsive to two procedures of TACE (defined as a "TACE non-responder" in Japan) [24].

Currently, there is no global standard definition of TACE failure and the treatment of TACE non-responders is controversial. A beneficial effect of HAIC with cisplatin for TACE non-responders was reported to be not inferior to sorafenib [52]. In contrast, another study indicated that sorafenib had a better disease control rate, longer time to progression and increased OS time in TACE non-responders compared with HAIC plus cisplatin [31]. Many studies have reported the beneficial effects of sorafenib for the treatment of TACE non-responders [32, 33, 52, 53]. However, continuing sorafenib therapy is not possible in most HCC patients because of progressive disease and impaired hepatic function. Therefore, some studies have investigated the use of alternative systemic therapies to follow sorafenib. A recent small study demonstrated that sorafenib therapy followed by TAI/HAIC induced partial responses and stable disease in some TACE non-responders [54].

Taken together, the findings from these studies and the results from the current retrospective study indicate that TACE non-responder patients with intermediate HCC may be candidates for sorafenib therapy followed by TAI/ HAIC. However, there is no good evidence for the effective simultaneous combination therapy of TACE and sorafenib [22].

Another consideration for TACE plus sorafenib therapy is the timing of administration. Following TACE, local concentrations of VEGF might become elevated, which might aid neoangiogenesis, a process implicated in the growth of HCC. Therefore, because sorafenib has antiangiogenic and antiproliferative effects, maintenance therapy for HCC often consists of TACE followed by sorafenib. Based on these effects, the administration of sorafenib after TACE is expected to inhibit the increase in VEGF signaling and prevent angiogenesis [25-28]. Furthermore, the inhibitory effect of TACE followed by sorafenib on tumor recurrence may extend the interval between TACE procedures [40]. Therefore, the correct timing for the initiation of sorafenib therapy after TACE is critical [22, 24, 46]. This is highlighted in a report by Kudo et al. [46], which indicated that starting sorafenib treatment too late after TACE did not affect the time to progression/recurrence, possibly because the pro-tumor effects of increased VEGF had already occurred. 
Most of the adverse events reported in the current study were of CTCAE Grade 1 or 2, and occurred in both groups. Only one Grade 3 event and one Grade 4 event were observed, both of which were liver dysfunction, indicating that TACE followed by sorafenib was well tolerated by Japanese TACE non-responder HCC patients. A previous study indicated that sorafenib was responsible for most adverse events [28] because they were similar to those observed in a sorafenib monotherapy trial. In that study, 23 of 35 patients discontinued treatment, of which 17 were due to adverse events and 2 were due to the patient's decision [28]. Therefore, potential adverse events might be managed by adjusting the dose of sorafenib.

This study had a number of limitations, including the relatively small number of non-randomized patients enrolled, the retrospective nature of the study, and the different treatment periods assessed; however, the safety and effectiveness results were in line with previous randomized trials.

\section{Conclusion}

In conclusion, this study demonstrated that even in patients with advanced HCC tumors and poor prognoses, the administration of TACE followed by sorafenib significantly improved the survival time and PFS in patients unresponsive to TACE, and was safe with few adverse events.

Acknowledgments The authors wish to thank J. Ludovic Croxford, $\mathrm{PhD}$ on behalf of Springer Healthcare Communications, for providing medical writing support, which was funded by Bayer Yakuhin, Ltd.

\section{Compliance with Ethical Standards}

Funding This study received no funding.

Informed consent Written informed consent was obtained from all patients when they began treatment but was not required for the present analyses because this was a retrospective study.

Conflict of interest Takamasa Ohki has received speaking fees from Otsuka Pharmaceutical Co., Ltd. The other authors have nothing to disclose.

Ethical approval Formal consent was not required for this type of study.

Open Access This article is distributed under the terms of the Creative Commons Attribution-NonCommercial 4.0 International License (http://creativecommons.org/licenses/by-nc/4.0/), which permits any noncommercial use, distribution, and reproduction in any medium, provided you give appropriate credit to the original author(s) and the source, provide a link to the Creative Commons license, and indicate if changes were made.

\section{References}

1. GLOBOCAN 2012 v1.0. Estimated cancer incidence, mortality, and prevalence worldwide. http://globocan.iarc.fr. Accessed 4 Jun 2015.

2. Lai CL, Ratziu V, Yuen MF, Poynard T. Viral hepatitis B. Lancet. 2003;362:2089-94.

3. Yuen MF, Hou JL, Chutaputti A. Asia Pacific Working Party on prevention of hepatocellular carcinoma. Hepatocellular carcinoma in the Asia Pacific region. J Gastroenterol Hepatol. 2009;24:346-53.

4. El-Serag HB. Epidemiology of hepatocellular carcinoma in USA. Hepatol Res. 2007;37:S88-94.

5. Mittal S, El-Serag HB. Epidemiology of hepatocellular carcinoma: consider the population. J Clin Gastroenterol. 2013;47:S2-6.

6. Umemura T, Ichijo T, Yoshizawa K, Tanaka E, Kiyosawa K. Epidemiology of hepatocellular carcinoma in Japan. J Gastroenterol. 2009;44:102-7.

7. Umemura T, Kiyosawa K. Epidemiology of hepatocellular carcinoma in Japan. Hepatol Res. 2007;37:S95-100.

8. Makuuchi M, Kokudo N, Arii S, Futagawa S, Kaneko S, Kawasaki S, Matsuyama Y, Okazaki M, Okita K, Omata M, Saida Y, Takayama T, Yamaoka Y. Development of evidencebased clinical guidelines for the diagnosis and treatment of hepatocellular carcinoma in Japan. Hepatol Res. 2008;38:37-51.

9. Omata M, Lesmana LA, Tateishi R, Chen PJ, Lin SM, Yoshida H, Kudo M, Lee JM, Choi BI, Poon RT, Shiina S, Cheng AL, Jia JD, Obi S, Han KH, Jafri W, Chow P, Lim SG, Chawla YK, Budihusodo U, Gani RA, Lesmana CR, Putranto TA, Liaw YF, Sarin SK. Asian Pacific Association for the Study of the Liver consensus recommendations on hepatocellular carcinoma. Hepatol Int. 2010;4:439-74.

10. Fattovich G, Stroffolini T, Zagni I, Donato F. Hepatocellular carcinoma in cirrhosis: incidence and risk factors. Gastroenterology. 2004;127:S35-50.

11. Nagaoki Y, Hyogo H, Aikata H, Tanaka M, Naeshiro N, Nakahara T, Honda Y, Miyaki D, Kawaoka T, Takaki S, Hiramatsu A, Waki K, Imamura M, Kawakami Y, Takahashi S, Chayama K. Recent trend of clinical features in patients with hepatocellular carcinoma. Hepatol Res. 2012;42:368-75.

12. Nishikawa H, Osaki Y. Non-B, non-C hepatocellular carcinoma. Int J Oncol. 2013;43:1333-42.

13. Llovet JM, Burroughs A, Bruix J. Hepatocellular carcinoma. Lancet. 2003;362:1907-17.

14. Ikai I, Arii S, Okazaki M, Okita K, Omata M, Kojiro M, Takayasu K, Nakanuma Y, Makuuchi M, Matsuyama Y, Monden M, Kudo M. Report of the 17th nationwide follow-up survey of primary liver cancer in Japan. Hepatol Res. 2007;37:676-91.

15. Tang ZY. Relapse and metastasis-a key point in research of primary hepatocarcinoma. Zhonghua Gandan Waike Zazhi. 1999;5:3-5.

16. Qiu LD, Ding YT. The research and therapy improvement of recurrence and metastasis of hepatocellular carcinoma. Gandan Waike Zazhi. 2001;9:6-7.

17. Bruix J, Sherman M. American Association for the Study of Liver Diseases. Management of hepatocellular carcinoma: an update. Hepatology. 2011;53:1020-2.

18. Cheng HY, Wang X, Chen D, Xu AM, Jia YC. The value and limitation of transcatheter arterial chemoembolization in preventing recurrence of resected hepatocellular carcinoma. World J Gastroenterol. 2005;11:3644-6.

19. Llovet JM, Real MI, Montaña X, Planas R, Coll S, Aponte J, Ayuso C, Sala M, Muchart J, Solà R, Rodés J, Bruix J. Barcelona 
Liver Cancer Group. Arterial embolisation or chemoembolisation versus symptomatic treatment in patients with unresectable hepatocellular carcinoma: a randomised controlled trial. Lancet. 2002;359:1734-9.

20. Lo CM, Ngan H, Tso WK, Liu CL, Lam CM, Poon RT, Fan ST, Wong J. Randomized controlled trial of transarterial lipiodol chemoembolization for unresectable hepatocellular carcinoma. Hepatology. 2002;35:1164-71.

21. Kudo M, Izumi N, Kokudo N, Matsui O, Sakamoto M, Nakashima O, Kojiro M, Makuuchi M, HCC Expert Panel of Japan Society of Hepatology. Management of hepatocellular carcinoma in Japan: Consensus-Based Clinical Practice Guidelines proposed by the Japan Society of Hepatology (JSH) 2010 updated version. Dig Dis. 2011;29:339-64.

22. Cheng AL, Amarapurkar D, Chao Y, Chen PJ, Geschwind JF, Goh KL, Han KH, Kudo M, Lee HC, Lee RC, Lesmana LA, Lim HY, Paik SW, Poon RT, Tan CK, Tanwandee T, Teng G, Park JW. Re-evaluating transarterial chemoembolization for the treatment of hepatocellular carcinoma: Consensus recommendations and review by an International Expert Panel. Liver Int. 2014;34:174-83.

23. Llovet JM, Peña CE, Lathia CD, Shan M, Meinhardt G, Bruix J. SHARP Investigators Study Group. Plasma biomarkers as predictors of outcome in patients with advanced hepatocellular carcinoma. J Hepatol. 2012;56:908-43.

24. Kudo M, Matsui O, Izumi N, Kadoya M, Okusaka T, Miyayama S, Yamakado K, Tsuchiya K, Ueshima K, Hiraoka A, Ikeda M, Ogasawara S, Yamashita T, Minami T, Liver Cancer Study Group of Japan. Transarterial chemoembolization failure/refractoriness: JSH-LCSGJ criteria 2014 update. Oncology. 2014;87: 22-31.

25. Sergio A, Cristofori C, Cardin R, Pivetta G, Ragazzi R, Baldan A, Girardi L, Cillo U, Burra P, Giacomin A, Farinati F. Transcatheter arterial chemoembolization (TACE) in hepatocellular carcinoma (HCC): the role of angiogenesis and invasiveness. Am J Gastroenterol. 2008;103:914-21.

26. Shim JH, Park JW, Kim JH, An M, Kong SY, Nam BH, Choi JI, Kim HB, Lee WJ, Kim CM. Association between increment of serum VEGF level and prognosis after transcatheter arterial chemoembolization in hepatocellular carcinoma patients. Cancer Sci. 2008;99:2037-44.

27. Wilhelm SM, Adnane L, Newell P, Villanueva A, Llovet JM, Lynch M. Preclinical overview of sorafenib, a multikinase inhibitor that targets both Raf and VEGF and PDGF receptor tyrosine kinase signaling. Mol Cancer Ther. 2008;7:3129-40.

28. Pawlik TM, Reyes DK, Cosgrove D, Kamel IR, Bhagat N, Geschwind JF. Phase II trial of sorafenib combined with concurrent transarterial chemoembolization with drug-eluting beads for hepatocellular carcinoma. J Clin Oncol. 2011;29:3960-7.

29. Wilhelm SM, Carter C, Tang L, Wilkie D, McNabola A, Rong H, Chen C, Zhang X, Vincent P, McHugh M, Cao Y, Shujath J, Gawlak S, Eveleigh D, Rowley B, Liu L, Adnane L, Lynch M, Auclair D, Taylor I, Gedrich R, Voznesensky A, Riedl B, Post LE, Bollag G, Trail PA. BAY 43-9006 exhibits broad spectrum oral antitumor activity and targets the RAF/MEK/ERK pathway and receptor tyrosine kinases involved in tumor progression and angiogenesis. Cancer Res. 2004;64:7099-109.

30. Llovet JM, Ricci S, Mazzaferro V, Hilgard P, Gane E, Blanc JF, de Oliveira AC, Santoro A, Raoul JL, Forner A, Schwartz M, Porta C, Zeuzem S, Bolondi L, Greten TF, Galle PR, Seitz JF, Borbath I, Häussinger D, Giannaris T, Shan M, Moscovici M, Voliotis D, Bruix J, SHARP Investigators Study Group. Sorafenib in advanced hepatocellular carcinoma. N Engl $\mathbf{J}$ Med. 2008;359:378-90.

31. Cheng AL, Kang YK, Chen Z, Tsao CJ, Qin S, Kim JS, Luo R, Feng J, Ye S, Yang TS, Xu J, Sun Y, Liang H, Liu J, Wang J, Tak
WY, Pan H, Burock K, Zou J, Voliotis D, Guan Z. Efficacy and safety of sorafenib in patients in the Asia-Pacific region with advanced hepatocellular carcinoma: a phase III randomised, double-blind, placebo-controlled trial. Lancet Oncol. 2009;10:25-34.

32. Ikeda M, Mitsunaga S, Shimizu S, Ohno I, Takahashi H, Okuyama H, Kuwahara A, Kondo S, Morizane C, Ueno H, Satake M, Arai Y, Okusaka T. Efficacy of sorafenib in patients with hepatocellular carcinoma refractory to transcatheter arterial chemoembolization. J Gastroenterol. 2014;49:932-40.

33. Ogasawara S, Chiba T, Ooka Y, Kanogawa N, Motoyama T, Suzuki E, Tawada A, Kanai F, Yoshikawa M, Yokosuka O. Efficacy of sorafenib in intermediate-stage hepatocellular carcinoma patients refractory to transarterial chemoembolization. Oncology. 2014;87:330-41.

34. Bruix J, Raoul JL, Sherman M, Mazzaferro V, Bolondi L, Craxi A, Galle PR, Santoro A, Beaugrand M, Sangiovanni A, Porta C, Gerken G, Marrero JA, Nadel A, Shan M, Moscovici M, Voliotis D, Llovet JM. Efficacy and safety of sorafenib in patients with advanced hepatocellular carcinoma: subanalyses of a phase III trial. J Hepatol. 2012;57:821-9.

35. Nishikawa H, Osaki Y, Iguchi E, Takeda H, Nakajima J, Matsuda F, Sakamoto A, Henmi S, Hatamaru K, Saito S, Nasu A, Kita R, Kimura T. Comparison of the efficacy of transcatheter arterial chemoembolization and sorafenib for advanced hepatocellular carcinoma. Exp Ther Med. 2012;4:381-6.

36. Cabrera R, Pannu DS, Caridi J, Firpi RJ, Soldevila-Pico C, Morelli G, Clark V, Suman A, George TJ Jr, Nelson DR. The combination of sorafenib with transarterial chemoembolisation for hepatocellular carcinoma. Aliment Pharmacol Ther. 2011;34:205-13

37. Qu XD, Chen CS, Wang JH, Yan ZP, Chen JM, Gong GQ, Liu QX, Luo JJ, Liu LX, Liu R, Qian S. The efficacy of TACE combined sorafenib in advanced stages hepatocellullar carcinoma. BMC Cancer. 2012;12:263.

38. Shao W, Zhang F, Cong N, Li J, Song J. Transarterial chemoembolization combined with sorafenib for advanced hepatocellular carcinoma. Oncol Lett. 2014;8:2263-6.

39. Erhardt A, Kolligs F, Dollinger M, Schott E, Wege H, Bitzer M, Gog C, Lammert F, Schuchmann M, Walter C, Blondin D, Ohmann C, Häussinger D. TACE plus sorafenib for the treatment of hepatocellular carcinoma: results of the multicenter, phase II SOCRATES trial. Cancer Chemother Pharmacol. 2014;74: 947-54.

40. Sansonno D, Lauletta G, Russi S, Conteduca V, Sansonno L, Dammacco F. Transarterial chemoembolization plus sorafenib: a sequential therapeutic scheme for HCV-related intermediatestage hepatocellular carcinoma: a randomized clinical trial. Oncologist. 2012;17:359-66.

41. Chao Y, Chung YH, Han G, Yoon JH, Yang J, Wang J, Shao GL, Kim BI, Lee TY. The combination of transcatheter arterial chemoembolization and sorafenib is well tolerated and effective in Asian patients with hepatocellular carcinoma: final results of the START trial. Int J Cancer. 2015;136:1458-67.

42. Liu L, Chen H, Wang M, Zhao Y, Cai G, Qi X, Han G. Combination therapy of sorafenib and TACE for unresectable HCC: a systematic review and meta-analysis. PLoS One. 2014;9:e91124.

43. Yang M, Yuan JQ, Bai M, Han GH. Transarterial chemoembolization combined with sorafenib for unresectable hepatocellular carcinoma: a systematic review and meta-analysis. Mol Biol Rep. 2014;41:6575-82.

44. Therasse P, Arbuck SG, Eisenhauer EA, Wanders J, Kaplan RS, Rubinstein L, Verweij J, Van Glabbeke M, van Oostero AT, Christian MC, Gwyther SG. New guidelines to evaluate the response to treatment in solid tumors. European Organization for Research and Treatment of Cancer, National Cancer Institute of 
the United States, National Cancer Institute of Canada. J Natl Cancer Inst. 2000;92:205-16.

45. NCI Common Terminology Criteria for Adverse Events (CTCAE) v.4 data files. http://evs.nci.nih.gov/ftp1/CTCAE/ About.html. Accessed 14 May 2015.

46. Raoul JL, Gilabert M, Piana G. How to define transarterial chemoembolization failure or refractoriness: a European perspective. Liver Cancer. 2014;3:119-24.

47. Raoul JL, Sangro B, Forner A, Mazzaferro V, Piscaglia F, Bolondi L, Lencioni R. Evolving strategies for the management of intermediate-stage hepatocellular carcinoma: available evidence and expert opinion on the use of transarterial chemoembolization. Cancer Treat Rev. 2011;37:212-20.

48. European Association For The Study Of The Liver; European Organisation For Research And Treatment Of Cancer. EASLEORTC clinical practice guidelines: management of hepatocellular carcinoma. J Hepatol. 2012;56:908-43.

49. Kondo M, Morimoto M, Ishii T, Nozaki A, Fukuda H, Numata K, Kobayashi S, Ohkawa S, Hidaka H, Nakazawa T, Shibuya A, Okuse C, Suzuki M, Sakamaki K, Morita S, Maeda S, Tanaka K. Hepatic arterial infusion chemotherapy with cisplatin and sorafenib in hepatocellular carcinoma patients unresponsive to transarterial chemoembolization: A propensity score-based weighting. J Dig Dis. 2015;16: 143-51.
50. Arizumi T, Ueshima K, Chishina $\mathrm{H}$, Kono M, Takita M, Kitai S, Inoue T, Yada N, Hagiwara S, Minami Y, Sakurai T, Nishida N, Kudo M. Validation of the criteria of transcatheter arterial chemoembolization failure or refractoriness in patients with advanced hepatocellular carcinoma proposed by the LCSGJ. Oncology. 2014;87:32-6.

51. Terashima T, Yamashita T, Arai K, Sunagozaka H, Kitahara M, Nakagawa H, Kagaya T, Mizukoshi E, Honda M, Kaneko S. Feasibility and efficacy of hepatic arterial infusion chemotherapy for advanced hepatocellular carcinoma after sorafenib. Hepatol Res. 2014;44:1179-85.

52. Kudo M, Imanaka K, Chida N, Nakachi K, Tak WY, Takayama T, Yoon JH, Hori T, Kumada H, Hayashi N, Kaneko S, Tsubouchi H, Suh DJ, Furuse J, Okusaka T, Tanaka K, Matsui O, Wada M, Yamaguchi I, Ohya T, Meinhardt G, Okita K. Phase III study of sorafenib after transarterial chemoembolisation in Japanese and Korean patients with unresectable hepatocellular carcinoma. Eur J Cancer. 2011;47:2117-27.

53. Nishikawa H, Kita R, Kimura T, Osaki Y. Transcatheter arterial embolic therapies for hepatocellular carcinoma: a literature review. Anticancer Res. 2014;34:6877-86.

54. Firouznia K, Ghanaati H, Alavian SM, Azadeh P, Nasiri Toosi M, Haj Mirzaian A, Najafi S, Shakiba M, Jalali AH. Transcatheter arterial chemoembolization therapy for patients with unresectable hepatocellular carcinoma. Hepat Mon. 2014;14:e25792. 